\title{
Carrier transport coefficients across GaAs-GaAlAs (100) interfaces
}

\author{
G. C. Osbourn and D. L. Smith \\ California Institute of Technology, Pasadena, California 91125
}

(Received 26 June 1979; accepted 22 August 1979)

\begin{abstract}
We present calculations of reflection and transmission coefficients for electrons and holes at (100) interfaces for the GaAs- $\mathrm{Ga}_{1-x} \mathrm{Al}_{x}$ As system. We consider semi-infinite crystals of the two semiconductors joined at an abrupt or compositionally graded interface. The calculations are performed using the empirical tight binding approximation. The transport coefficients were computed as a function of the components of the incident carrier wavevector normal and parallel to the interface. We have investigated the transport coefficients for incident states near various band minima into different final state channels. The transmission into states with qualitatively similar character to the incident state is found to be much greater than transmission into states of different character. For example, an electron near the $X$ minimum normal to the interface in $\mathrm{Ga}_{1-x} \mathrm{Al}_{x} \mathrm{As}$ transmits into the $X$ valley of $\mathrm{GaAs}$ with much greater probability than it transmits into the $\Gamma$ minimum of GaAs. We have investigated the dependence of the transport coefficients on alloy composition. The effect of compositional grading of the interface on the transport coefficients has also been investigated.
\end{abstract}

PACS numbers: 73.40.Lq, 72.80.Ey, 71.25.Cx

\section{INTRODUCTION}

Molecular beam epitaxy techniques have made possible the production of well-characterized semiconductor-semiconductor interfaces which are very nearly atomically abrupt. ${ }^{1}$ The GaAs- $\mathrm{Ga}_{1-x} \mathrm{Al}_{x}$ As system is one that has often been studied. At an interface between two semiconductors the change in crystal potential can result in the quantum mechanical reflection of electrons and holes even if the carriers have enough energy to cross the interface. Here we present a calculation of electron and hole transmission and reflection coefficients for abrupt and compositionally graded (100) $\mathrm{GaAs}-\mathrm{Ga}_{1-x} \mathrm{Al}_{x}$ As interfaces. Our model consists of two semi-infinite semiconductor crystals treated in the empirical tight-binding method. The eigenstate of the interface system contains an incident Bloch function, reflected and transmitted Bloch functions, and exponentially decaying evanescent states. ${ }^{2,3}$ The transport coefficients are obtained from the amplitudes of the transmitted and reflected Bloch states and the group velocity of these states.

The paper is organized as follows. In Sec. II we discuss the theoretical methods used in the calculation. Section III contains the qualitative features of our results. The detailed numerical results are presented in Sec. IV, and we present our conclusions in Sec. V.

\section{THEORETICAL METHOD}

The problem is to construct eigenstates of the interface system which represent the transmission and reflection of an incoming free carrier at the interface. We carry this out using a linear combination of states of the bulk materials with the same energy $E$. Because the crystal is periodic in the plane parallel to the interface, $k$ parallel to the interface $(k)$ is a good quantum number. Since $\mathbf{k}^{\|}$is conserved, we need in- clude only those bulk states that have the same component $\mathbf{k}^{\|}$ as the incoming state, but any component of $k$ normal to the interface $\left(\mathbf{k}^{\perp}\right)$ that corresponds to a state of energy $E$. These $\mathbf{k}^{\perp}$ can in general be real or complex valued, with the real $\mathbf{k}^{\perp}$ producing Bloch states and the complex $\mathbf{k}^{\perp}$ producing evanescent states. ${ }^{2,3}$ The evanescent states are bulk eigenstates that exponentially grow in some direction. In the bulk material they do not satisfy the boundary conditions and are not allowed. In the interface system those evanescent states that exponentially grow in the normal direction towards the interface are allowed and must be included in the solution. Evanescent states do not carry flux normal to the interface, so that all contributions to the reflection and transmission of carriers comes from the Bloch states. Our solution takes the form

$$
\Psi= \begin{cases}\Phi_{k_{l}}^{E} \|, k_{I} \perp \\ \sum_{n, i} b_{n}\left(R_{i}\right) \sum_{\alpha} A_{\alpha}\left(r-\mathbf{R}_{i}\right) \exp i \mathbf{k} \psi \cdot R_{k_{I}} \|, k_{1 \alpha} \perp & \text { side 1 } \\ \sum_{\alpha} B_{\alpha} \phi_{k_{I}}^{E} \|_{, k_{2 \alpha} \perp} & \text { graded region }\end{cases}
$$

where ${ }_{k_{I}}^{E} \|, k_{I} \perp$ is the incident Bloch state (which carries flux towards the interface) with energy $E$ and $\mathbf{k}_{I}=\mathbf{k}_{I} \|+\mathbf{k}_{I}{ }^{\perp}$, $\phi_{k_{l} \|, k_{1 \times} \perp}^{E}$ and $\phi_{k_{l} \|, k_{2 \alpha} \perp}^{E}$ are the outgoing Bloch states which carry flux away from the interface (real $k_{1, k}^{\perp}$ or $k_{2 k}^{\perp}$ ) or decaying evanescent states (complex $k_{1 / k}^{\perp}$ or $\left.k_{2, k}^{\perp}\right), F_{n}\left(r-R_{i}\right.$ ) is an atomic function of type $n=\left(s, p_{x}, p_{y}, p_{z}\right)$ centered on atomic site $R_{i}$, $A_{c r}$ and $B_{\alpha}$ are bulk state expansion coefficients, and $b_{n}\left(R_{i}\right)$ is the atomic expansion coefficient for $F_{n}\left(r-R_{i}\right)$ in the compositionally graded region. The eigenstate is determined 
by solving the Schrodinger equation for the expansion coefficients $A_{c}, B_{w}$, and $b_{n}\left(R_{i}\right)$.

The Bloch and evanescent states for the two bulk materials are obtained in a tight-binding form, using one $s$ and three $p\left(p_{x}, p_{y}, p_{z}\right)$ type atomic orbitals per atomic site. In particular, we take ${ }^{4}$

$$
\phi_{k \|, k \perp}^{E}=\sum_{n, i} C_{n}^{\mathbf{k}}\left(\mathbf{R}_{i}\right) e^{i \mathbf{k} \cdot \mathbf{R}_{i}} F_{n}\left(r-\mathbf{R}_{i}\right)
$$

where $\phi_{k \|, k \perp}^{E}$ is the Bloch or evanescent state of energy $E, F_{n}(r$ $\left.-R_{i}\right)$ is the atomic orbital of type $n=\left(s, p_{x}, p_{y}, p_{z}\right)$ at site $\mathbf{R}_{i}$, and the $C_{n}^{\mathbf{k}}\left(\mathbf{R}_{i}\right)$ are atomic expansion coefficients. The $C_{n}^{\mathbf{k}}\left(\mathbf{R}_{i}\right)$ are found in the usual way by diagonalizing the tight-binding matrix for the $\mathbf{k}$ that correspond to the energy $E$ :

$$
\left\langle F_{j}\left(r-R_{l}\right)|H-E| \phi_{k \|, k \perp}^{E}\right\rangle=0
$$

The tight-binding parameters in the tight-binding matrix characterize the bulk materials and are inputs to the calculation. Our treatment of these parameters for GaAs, AlAs, and $\mathrm{Ga}_{1-x} \mathrm{Al}_{x}$ As is discussed at the end of this section.

The coefficients $A_{\alpha}, B_{\alpha}$, and $b_{n}\left(R_{i}\right)$ are determined by taking the overlap of the one electron Schrodinger equation |using $\Psi$ from Eq. (1)] with all of the atomic orbitals in the crystal:

$$
\left\langle F_{j}\left(r-R_{1}\right)|H-E| \Psi\right\rangle=0
$$

This produces an infinite set of equations; however, all but a finite number of them are automatically satisfied by construction. For atomic orbitals in the bulk materials that do not have overlaps with orbitals in the graded region or on the other side of the interface, Eq. (4) reduces to a linear combination of terms of the form in Eq. (3), so Eq. (4) is automatically satisfied. Only overlaps from orbitals in a finite number of atomic planes parallel to the interface at or near the graded region needed be considered. The periodicity of the crystal in these parallel planes further limits the number of orbitals in these planes that need be considered to a finite set. This results in an $N \times N$ set of linear inhomogeneous equations to solve for the expansion coefficients, with the inhomogeneous parts due to the incoming state in Eq. (1).

Once the expansion coefficients $A_{c k}$ and $B_{r r}$ have been found for a given incident Bloch state, the transport coefficients for the reflection and transmission Bloch states are found using the expressions

$$
\begin{aligned}
& R_{c \alpha}=\left|A_{\alpha}\right|^{2}\left|\frac{\partial E_{\alpha}}{\partial k \perp}\right| /\left|\frac{\partial E_{I}}{\partial k \perp}\right| \\
& T_{\beta}=\left|B_{\beta}\right|^{2}\left|\frac{\partial E_{\beta}}{\partial k \perp}\right| /\left|\frac{\partial E_{I}}{\partial k^{\perp}}\right|
\end{aligned}
$$

where $\left|\partial E_{\alpha} / \partial k^{\perp}\right|$ is the group velocity for the Bloch state $\alpha$ normal to the interface. The transport coefficients satisfy the conservation of flux relation

$$
\sum_{\alpha} R_{\alpha}+\sum_{\beta} T_{\beta}=1
$$

The tight-binding parameters used in the calculation were obtained as follows: The GaAs and AlAs parameters were chosen to fit the bulk band structures to pseudopotential re- sults. 5,6 We include empirical valence band offsets for the GaAs-GaAlAs system equal to $15 \%$ of the direct gap differences between GaAlAs and GaAs. ${ }^{7}$ The parameters so obtained will be presented elsewhere. ${ }^{8}$ The $\mathrm{Ga}_{1-x} \mathrm{Al}_{x} \mathrm{As}$ alloy parameters are taken to be weighted averages of the GaAs and AlAs parameters. This virtual crystal type approximation produces band structures that vary linearly with alloy concentration $x$.

The atomic planes in the compositionally graded region between the $\mathrm{GaAs}$ and $\mathrm{Ga}_{1-x} \mathrm{Al}_{x}$ As bulk regions are treated as alloys of composition $x^{\prime}$, where $x^{\prime}$ varies linearly from zero at the GaAs side to $x$ at the $\mathrm{Ga}_{1-x} \mathrm{Al}_{x} \mathrm{As} \mathrm{Ga}_{1-x} \mathrm{Al}_{x}$ As side. The overlaps of $\mathrm{Ga}_{1-x^{\prime}} \mathrm{Al}_{x^{\prime}}$ orbitals with second nearest neighbor $\mathrm{Ga}_{1-x^{\prime \prime}} \mathrm{Al}_{x^{\prime \prime}}$ orbitals are taken as a weighted average of $\mathrm{Ga}-\mathrm{Ga}$, $\mathrm{Al}-\mathrm{Al}$, and $\mathrm{Ga}-\mathrm{Al}$ overlaps. We take the $\mathrm{Ga}-\mathrm{Al}$ overlap to be the average of the $\mathrm{Ga}-\mathrm{Ga}$ and $\mathrm{Al}-\mathrm{Al}$ second nearest neighbor overlap.

\section{QUALITATIVE FEATURES OF CARRIER TRANSPORT COEFFICIENTS}

The case of interest is that of incoming Bloch states near a band extrema point (conduction band minimum for electrons, valence band maximum for holes). The transport coefficients for the outgoing transmission and reflection Bloch states will depend on the alloy concentration $x$, incident wavevector, and the size of the compositionally graded region.

First we note that incident states from one side of the interface only need be considered because the transmission coefficient from an incident state with wavevector $k_{I}$ to a state of wavevector $\mathbf{k}_{0}$ is the same as that for the reciprocal case of an incident state with wavevector $-\mathbf{k}_{0}$ transmitting to a state with wavevector $-\mathbf{k}_{I}{ }^{8}$ :

$$
T\left(\mathbf{k}_{I}, \mathbf{k}_{0}\right)=T\left(-\mathbf{k}_{0},-\mathbf{k}_{I}\right)
$$

Many of the qualitative features of the transport coefficients can be summarized by the result that large transport into a given outgoing state corresponds to the degree to which that state qualitatively resembles the incident state. Consider the threshold behavior of the transport coefficients. For incident states with $\mathbf{k}_{I}$ approaching a critical point $\mathbf{k}_{C}$, there is a reflection state with wavevector $\mathbf{k}_{R}$ that also approaches $\mathbf{k}_{C}$. These states become more similar as $\mathbf{k}_{I}$ approaches $\mathbf{k}_{C}$, so that reflection into the $\mathbf{k}_{R}$ state becomes large near $\mathbf{k}_{C}$. In fact, reflection into the $\mathbf{k}_{R}$ state must approach unity linearly as $\mathbf{k}_{R}$ approaches $\mathbf{k}_{C} .{ }^{8} \mathrm{At}$ a given $k_{I}$, the relative importance of the various transmission states (if there is more than one) is also determined by their qualitative similarity to the incident state. For example, incident electrons near the $X$ minimum (normal to interface) in $\mathrm{Ga}_{1-x} \mathrm{Al}_{x}$ As can transmit to states near the $X$ or I' minima in GaAs. The transmitted state near $X$ in $\mathrm{GaAs}$ is qualitatively similar to the incident state while the state near $\Gamma$ ' in GaAs is not. The transport into the state near $X$ in GaAs is found to be much larger than that into the state near $\Gamma$.

The alloy composition $x$ determines how different $\mathrm{Ga}_{1-x^{-}}$ $\mathrm{Al}_{x}$ As is from GaAs, ' with large $x$ corresponding to larger differences. Thus for fixed $k_{I}$, large $x$ corresponds to a larger difference in crystal potentials and greater reflection probability for the incident carrier.

Finally, the number of atomic layers over which the in- 


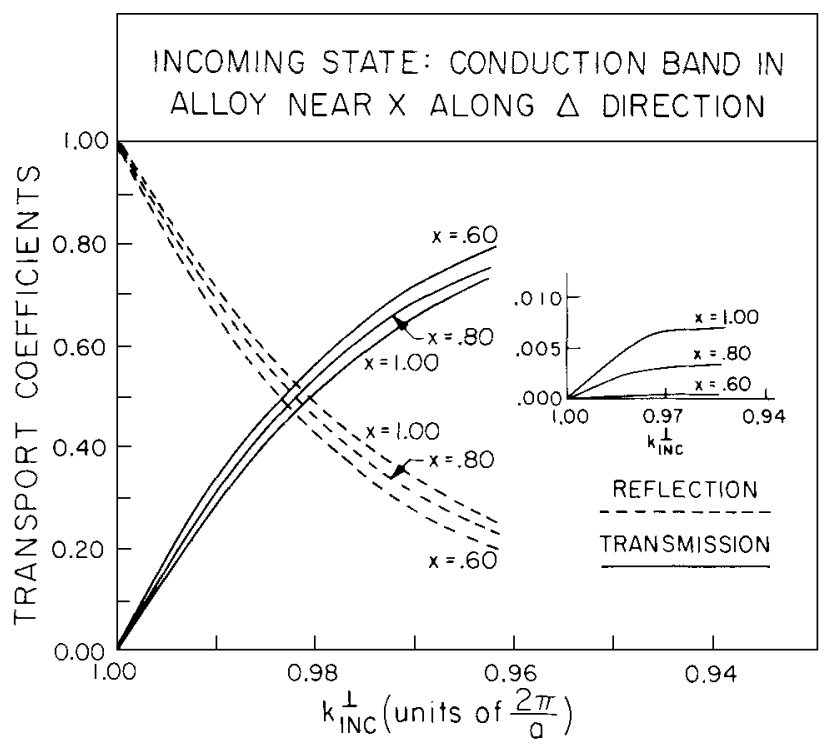

FIG. 1. Transport coefficients as a function of incident wavevector normal to the interface for different alloy compositions. In our calculation the alloy is indirect for the range of compositions in the figure. The incident states are in the alloy near the $X$ conduction band minimum along the $\Delta$ direction normal to the interface. The central portion of the figure shows the transport coefficients into states near the corresponding $X$ minima. The inset shows the transmission coefficients into states near the $\Gamma$ minimum of GaAs.

terface is graded determines (for large number of layers) how smoothly the crystal potential changes from GaAs to $\mathrm{Ga}_{1-x} \mathrm{Al}_{x}$ As. Smoother transitions lead to less reflection for fixed incident states, so that for sufficiently many layers, increasing the layer number increases transmission.

\section{RESULTS}

The results presented in this section are for incoming $\mathrm{Ga}_{1-x} \mathrm{Al}_{x}$ As states at (100) interfaces. In Fig. (1) electron

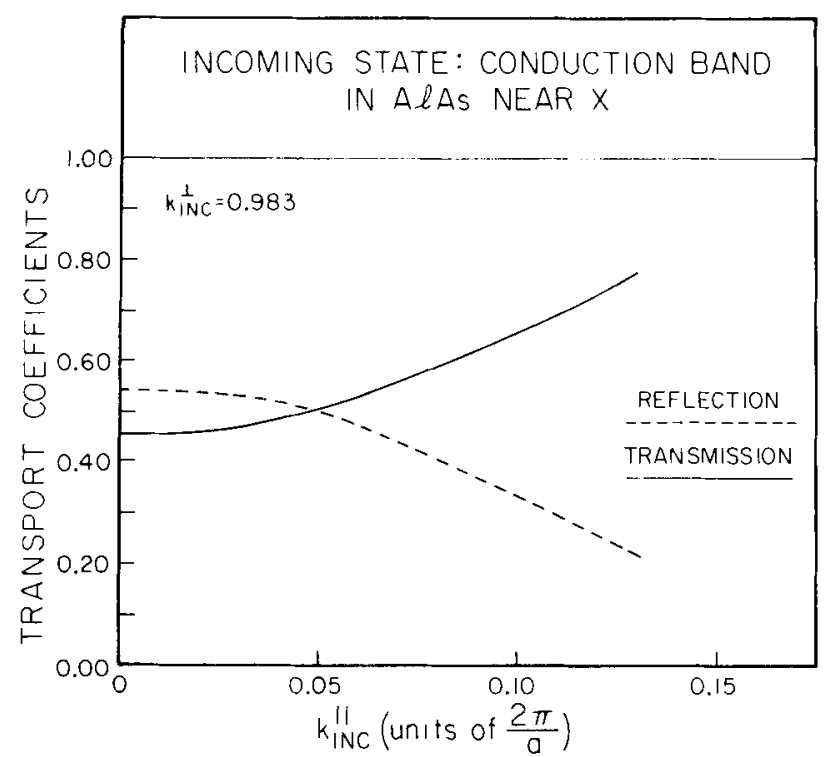

FIG. 2. Transport coefficients as a function of the incident wavevector component parallel to the interface. The incident states are in AlAs near the perpendicular $X$ conduction band minimum. The states have fixed $k_{\mathrm{INC}}{ }^{\perp}$ equal to $2 \pi / a(0.983,0,0)$. The parallel component of the incident wavevector is in the $V$ direction.

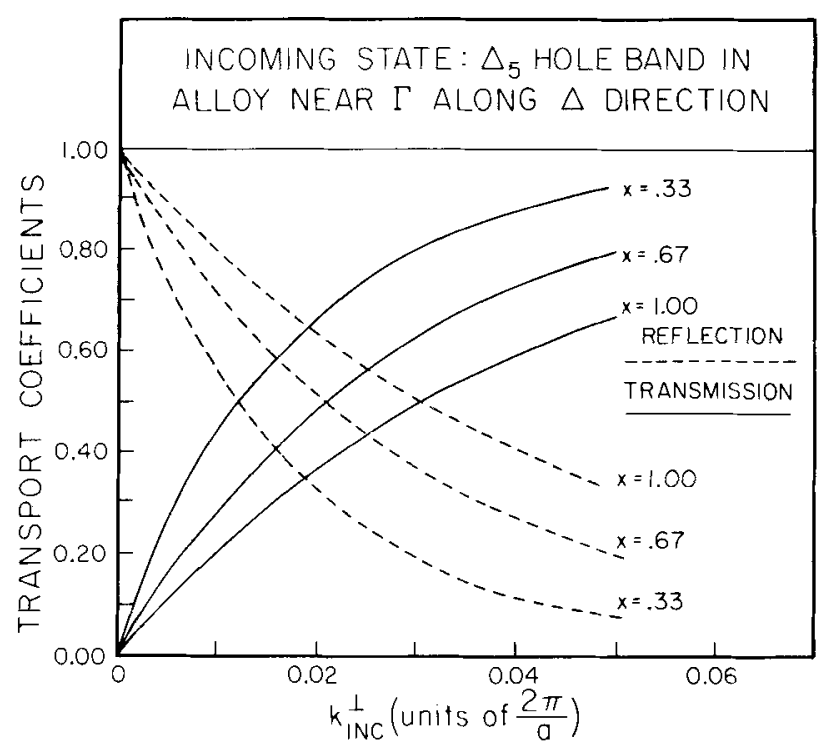

FIC. 3. Transport coefficients as a function of incident wavevector normal to the interface for different alloy compositions. The incident states are in the alloy near the $\Gamma$ valence band maxima along the $\Delta$ direction normal to the interface. All states involved have $\Delta_{5}$ symmetry.

transport coefficients are presented for a range of alloy concentrations in which the alloy is indirect. Transport into GaAs states near $X$ is presented in the central figure, and the much smaller transport into states near $\Gamma$ is presented in the inset. The curves in Fig. (2) show the dependence of the transport coefficients on $\mathbf{k}^{\|}$for fixed $\mathbf{k}^{\perp}$ for incident AlAs states near $X$. The dependence of the transport coefficients on $\mathbf{k}^{\|}$is observed to be much weaker than on $\mathbf{k}^{\perp}$. In Fig. (3) we present $\Delta_{5}$ hole transport coefficients. Although a detailed treatment

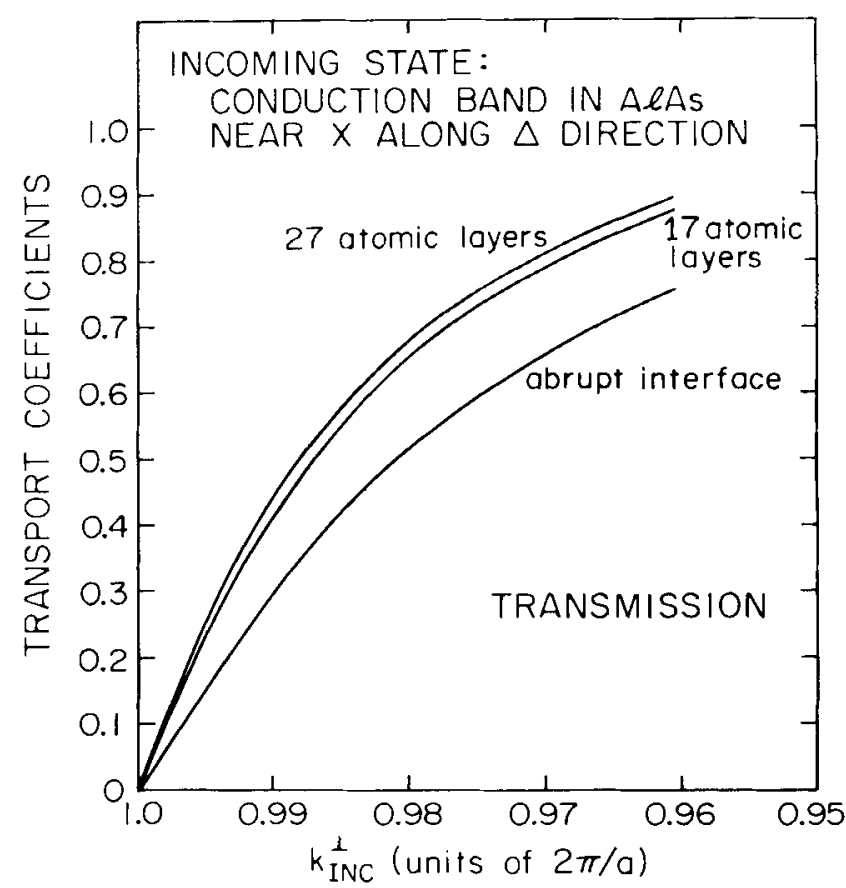

FIG. 4. Transmission coefficients as a function of incident wavevector normal to the interface for abrupt and compositionally graded interfaces. The incident states are in AlAs near the $X$ conduction band minimum along the $\Delta$ direction normal to the interface. The graded interface transmission coefficients are shown for interfaces graded over 17 and 27 atomic layers. 
of hole transport should include the spin orbit splitting, this interaction will not alter the qualitative features of the result shown here. The curves in Fig. (4) illustrate the dependence of the transport coefficients on the number of atomic layers in the compositionally graded interface region.

\section{SUMMARY AND CONCLUSIONS}

We have presented reflection and transmission coefficients for electrons and holes at abrupt and graded (100) GaAs$\mathrm{Ga}_{1-x} \mathrm{Al}_{x} \mathrm{As}$ interfaces. We find that transport into states that are qualitatively similar to the incident states is much greater than transport into states of different character. For example, electrons in $\mathrm{Ga}_{1-x} \mathrm{Al}_{x}$ As near $X$ preferentially transport into states near $X$ in GaAs over states near $\Gamma$. The threshold dependence and the alloy concentration dependence of the transport coefficients has been determined. For electrons in a particular valley we find the transport coefficients to depend less sensitively on $\mathbf{k}_{I}^{\|}$than on $\mathbf{k}_{I}{ }^{\perp}$. Finally we have determined the dependence of the transport coefficients on compositional grading of the interface.

\section{ACKNOWLEDGMENT}

The authors acknowledge many useful conversations with J. N. Schulman and T. C. McGill. This work was supported in part by the Department of Energy. One of us (DLS) acknowledges receipt of a fellowship from the Alfred P. Sloan Foundation.

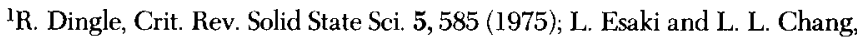
Crit. Rev. Solid State Sci. 6, 195 (1976); and references contained therein.

${ }^{2}$ V. Heine, Proc. Phys. Soc. 81, 300 (1963).

${ }^{3}$ E. I. Blount, Solid State Phys. 13, 305 (1962).

${ }^{4}$ J. C. Slater and G. F. Koster, Phys. Rev. 94, 1498 (1954).

${ }^{5}$ J. R. Chelikowsky and M. L. Cohen, Phys. Rev. B 14, 556 (1976).

${ }^{6}$ F. Hess, I. Topol, K. R. Schulze, H. Newmann, and K. Unger, Phys. Status Solidi (b) 55, 187 (1973)

${ }^{7}$ R. Dingle, W. Wiegmann, and C. H. Henry, Phys. Rev. Lett. 33, 827 (1974).

${ }^{8}$ G. C. Osbourn and D. L. Smith, Phys. Rev. B 19, 2124 (1979) 